\title{
COOPERAR DESDE LA DIVERSIDAD
}

\author{
Óscar Cristi* \\ Eugenio Cáceres ${ }^{* *}$
}

* Rector de la Universidad de los Andes.

** Director de Relaciones Internacionales de la Universidad de los Andes. 


\section{RESUMEN}

En el contexto de la globalización, como fenómeno cultural, se insertan hoy las universidades como empresas del conocimiento que buscan la cooperación entre ellas y también con otras instituciones de los sectores cultural, social y económico, en el entendimiento básico de que su tarea principal es el saber y el servicio a la sociedad.

El análisis identifica la diversidad institucional como uno de los fundamentos de la colaboración universitaria y consigna sus características generales y específicas, en referencia a las experiencias regionales y locales de los países europeos y de los EE.UU. de Norteamérica. Se enumeran también los "factores de protección" y "factores de riesgo" de las acciones cooperativas y se plantean algunas apreciaciones respecto a lo que está sucediendo en el país en torno a la cooperación universitaria.

\section{ABSTRACT}

Within globalization context, universities seek to cooperate among themselves and with other cultural, social and economic institutions, although always keeping in mind that their main task is knowledge and support to society.

This analysis identifies institutions' diversity as one of the reasons for universities' collaboration, and establishes its characteristics related to experiences in Europe and in the United States. It also points out "protection agents" and "risk agents" for the cooperative actions and it raises some issues on what is happening in Chile around universities' cooperation. 


\section{COOPERAR DESDE LA DIVERSIDAD}

El contexto

Estamos insertos en un proceso de globalización que crece día a día, aunque con distintos ritmos de desarrollo y avance según sea la materia sobre la cual se haga el análisis. Es alto el acceso a los bienes simbólicos -información, comunicación y expresión- pero escaso respecto de los económicos y aún menor de los culturales; amplio en el menú de imágenes, bastante más restringido en los del progreso material y modos culturales. Las redes del saber crecen cada vez con mayor amplitud, cobertura y respeto por las diferencias, aunque se generen ciertas tensiones entre la creciente expansión de los mensajes ajenos y la búsqueda de autonomías y espacios propios. La globalización nos coloca frente a una diversidad de culturas, sensibilidades y diferencias que nos hacen recrear las nuestras a través de las ajenas. Junto al respeto multicultural se produce la consecuente autorrecreación.

El capital -ya no sólo físico y financiero sino que también humano, organizacional y social-, es altamente móvil y atraído por el trabajo calificado. Esta característica es especialmente notoria en las "empresas del conocimiento", como son las instituciones de educación superior, específicamente las universidades. Ellas no sólo enseñan y difunden el conocimiento sino que, fundamentalmente, lo crean, ordenan y sistematizan, y lo hacen disponible para el medio social en que se insertan.

Con la globalización se adquiere conciencia de que la soledad es marginalidad y de que el conocimiento, como elemento característico de nuestra sociedad, es objeto relevante de comunicación e interacción. 
Asimismo, el trabajo conjunto produce resultados positivos y enriquece la diversidad; crecen las redes del saber cada vez más con mayor amplitud y cobertura, y con respeto a las diferencias.

Por ello, y en especial en el ámbito universitario, la cuestión principal ya no se refiere a si ejercer o no la cooperación, sino a la más adecuada forma de hacerlo.

\section{¿Por qué cooperar?}

Se puede profundizar en esta conclusión revisando brevemente cuatro razones esenciales, a la vez que complementarias, que hacen necesaria y conveniente la cooperación universitaria.

En primer lugar, ésta encuentra su más profunda raíz en el convencimiento de que la promoción de la enseñanza superior es una tarea común que exige el fomento de las relaciones de intercambio y de mutua ayuda, marcando así una estrecha colaboración que ha de reinar siempre en el campo de la cultura. Sin embargo, esta razón de ser no se limita a la cooperación entre instituciones universitarias, sino que se manifiesta también en su afán por colaborar con otras organizaciones que conforman el entramado social.

La universidad es un lugar para compartir conocimientos, debates y actitudes críticas y puede entenderse como un proyecto que versa sobre un conocimiento no cerrado en sí mismo, sino abierto a las tareas con que las personas contribuyen a la sociedad y a las formas con que los ciudadanos se comunican y participan en el proceso de organización social y cultural. El rol de la universidad es el de un servicio para los individuos y para la comunidad -así se estableció desde su origen-, y es éste el parámetro con que ha de determinarse su calidad y usarse como guía principal de sus iniciativas, planes, programas y proyectos.

Del mismo modo, es la calidad académica, como resultante de la integración de múltiples y distintos factores, el elemento que asegura el futuro institucional en la enseñanza e investigación, en términos de su apertura y relación con otras instituciones similares. Se tienen en 
cuenta, además, variables políticas, sociales y financieras que pueden cambiar, facilitar o dificultar las tareas propias, así como el criterio y la habilidad para cooperar nacional e internacionalmente.

En segundo lugar, esta pujante sociedad del conocimiento muestra otras dimensiones a las cuales la educación superior debe adecuarse (Simonyi, 1999). Si el saber es universal y los límites políticos, ideológicos y geográficos ya no son barreras para establecer comunidad de ideas, surge, como natural consecuencia, la necesidad de comunicación y de acentuada cooperación entre las universidades en los diversos niveles en que se planteen, sea en lo regional, nacional, intercontinental o mundial. Tal cooperación se entiende, casi, como un deber a cumplir, pues el mejor lugar para trabajar con el conocimiento es la universidad, eso sí en la oportunidad y complementariedad adecuadas.

En tercer lugar, el siempre diverso desarrollo de estas instituciones exige que busquen la cooperación nacional e internacional porque, en la medida en que la complementariedad es más obvia, la ayuda mutua y la colaboración horizontal resulta efectivamente enriquecedora. Es conveniente que cada universidad coopere con otras en sus características complementarias y como parte de programas de distinto plazo. Incluso la mejor de ellas no lo es en todas las áreas que cubre y la menos desarrollada integralmente suele tener unidades académicas específicas de alta calidad.

En cuarto lugar, esta colaboración está también exigida por la limitación de recursos materiales y humanos presente en muchas entidades, agravada por el inadecuado y disparejo financiamiento estatal. Estas limitaciones impiden que una universidad pretenda abordar por completo todos los aspectos vinculados al desarrollo y transmisión de la ciencia. La antigua y aún vigente duda acerca de la definición de estrategias de cooperación o de competencia se resuelve hoy, con certeza, a favor de la primera. A ello contribuyen, en buena parte, recursos disponibles que se asignan, preferentemente, a consorcios en que las universidades se suman a otras, a empresas estatales y privadas, o a organismos públicos interesados, identificándose lo que varios autores denominan la triple alianza a la que concurren los sectores educacional, laboral y estatal (Reed, 2000). 
En definitiva, tal como lo señala Llano (1985), “...la innovadora reposición de la universidad, debe concentrarse en destacar el carácter universal del trabajo universitario, y hacerlo posible mediante un creciente esfuerzo de integración de personas y centros en una dinámica común". La caracterización universitaria vigente (Fermoso, 1993), incluye, junto a los elementos tradicionales, la creciente colaboración con la empresa y la consolidación de la tendencia hacia la cooperación nacional e internacional, en parte originada en el incremento de la investigación como una actividad no exclusiva de la universidad. El desarrollo de redes de investigadores que se comunican efectivamente, pese a las largas distancias, contribuye a la innovación científica y tecnológica del desarrollo.

Sin duda, ha habido un evidente crecimiento de la cooperación entre las universidades en la última década. Por ello, es urgente la ejecución de proyectos de colaboración que mejoren la calidad de la acción universitaria, que adopten formas de mayor cooperación interregional, configurando, quizás, determinadas áreas académicas y fortaleciendo las relaciones entre el sistema educacional, el entrenamiento intelectual, la investigación y el avance tecnológico.

¿Cómo se relacionan la diversidad con la cooperación?

En la actual sociedad del conocimiento no existe una sola universidad. El mundo universitario prueba la coexistencia de varios modelos que corresponden a situaciones sociales complejas y distintas, y a ideas económicas, políticas y culturales diferentes en países y continentes diversos.

Teniendo presente que las responsabilidades universitarias son idénticas en todo el mundo, sí cabe considerar que son heterogéneos sus grados de desarrollo y que su mejoramiento pasa, necesariamente, por la buena formación y entrenamiento de sus académicos, y por la incorporación a programas cooperativos de alta calidad.

La colaboración entre universidades no sólo tiene un impacto positivo sobre el crecimiento de instituciones heterogéneas, sino que, esta misma diversidad, alienta la cooperación, reconociéndose su importancia para el desarrollo de la sociedad. Respecto a este punto, 
Anderson (1999) transcribe el comentario de Krasno, ex Presidente del Instituto Internacional de Educación, en el sentido de que la cooperación e intercambios educacionales "...construyen el capital humano requerido para combatir la pobreza y manejar el complejo problema de la modernización, desarrollando un diálogo informado e internacional, unido a un gran entendimiento mutuo...", el que crea "...lazos perdurables entre individuos e instituciones... que, fomentan la aparición de sociedades creativas, multilaterales y toman ventaja del amplio rango de talentos intelectuales y creativos a nivel mundial. El intercambio educacional, por su propia naturaleza, es el medio principal para cumplir estas metas".

En el tema del trabajo interinstitucional hay una larga experiencia europea. Ya en declaraciones y documentos de la década de los '50 (París 1953) se planteaban, para las universidades europeas, ciertas modalidades de equivalencias y movilidad; en la de los '70, algunas de ellas se extendían a veintiún estados miembros abarcando temas como: organización de estudios comunes, relaciones entre investigación y sectores productivos, y la experiencia británica de los split-programs en posgrados, entre otros. Hoy existen diversas organizaciones supranacionales activas, en el sentido práctico, de cooperación universitaria, intercambios académicos y reconocimiento internacional. Cabe destacar los acuerdos atingentes a educación, cultura, ciencia y tecnología ligados a los tratados comerciales que Chile ha suscrito y está por suscribir, que abren nuevas y más amplias posibilidades de asociación y colaboración, todas ellas en la perspectiva del mejoramiento cualitativo.

Asimismo, en el caso de los Estados Unidos de Norteamérica, existe experiencia acumulada respecto del tema que, en su caso, toma una amplia diversidad de tipologías, en especial en lo pertinente a relacionar diversos tipos institucionales, como es el caso de las universidades con los community colleges (Fincher, 2002), asunto que en Chile se planteó como una característica posible del sistema que se inició a comienzos de los ' 80 , pero aún con escasa aplicación.

En el progreso de la cooperación entre instituciones, de por sí heterogéneas, juega un rol preponderante la existencia de ciertos estándares de calidad de aceptación general. El diálogo informado, 
entre aquellas interesadas en enfrentar desafíos en conjunto, resulta indispensable a la hora de evaluar las fortalezas y las ventajas comparativas de cada una, de modo de lograr una complementariedad con beneficios netos por sobre la alternativa de la acción independiente. Con todo, la aplicación de estos estándares, sea a través de sistemas de acreditación u otros, no puede en ningún caso desincentivar la diversidad, puesto que, sin ella, no hay colaboración y sí, en cambio, dilapidación de recursos y pérdida de una excelente oportunidad de incremento de la creatividad.

\section{Las bases de la cooperación}

Aun cuando resulta obvio que cada experiencia de cooperación se ejerce según sus propias condiciones, la descripción y explicación del plan implementado por trece instituciones a partir de 1988 -en lo que se ha denominado el University of Maryland System (UMS) (Langenberg, 1994)-, permite identificar ciertos factores que, de una u otra manera, están o debieran estar presentes en cualquier acción de cooperación universitaria. Se señalan cinco componentes que proporcionan una mejor comprensión y juicio sobre la eficacia de estos sistemas: sinergia, estrategia, eficiencia, responsabilidad social (traducción quizás no plenamente acertada de la expresión inglesa accountability) e integridad.

El factor "sinergia" alude a la existencia en todo sistema de un potencial enorme para aumentar, y aun transformar, el desempeño de instituciones individuales mediante un esfuerzo coordinado. Esta sinergia impacta en los actores y en los territorios en que se sitúan las entidades. Para los estudiantes, significa aumentar las oportunidades y opciones académicas en sus curricula, y en la utilización de distintos servicios de apoyo, como sistemas integrados de bibliotecas, instalaciones deportivas y residencia; para los académicos, cotejar sus experiencias con las de otros, y conocer y efectuar sus proyectos de investigación en laboratorios de todos los participantes. Para los territorios, representa la posibilidad de utilizar la fuerza intelectual combinada de múltiples organismos para mejorar aspectos del proceso integral: económicos, ambientales, cuidado de la salud, educativos y pertinentes a otras necesidades de servicio público. Uno de los tantos ejemplos es el esfuerzo conjunto realizado por once instituciones del 
UMS para mejorar la educación básica y media en matemática y ciencias, que logró un subsidio especial por US\$ 6 millones por parte de la Fundación Nacional de las Ciencias de los EE.UU. de Norteamérica.

El factor "estrategia" apunta a la determinación de metas comunes, a la identificación y asignación de roles complementarios a las instituciones componentes del UMS y a la coordinación de las tácticas que se empleen. Esto supone que los miembros del sistema logran mejor los objetivos individuales que si los trabajaran como metas superpuestas. La obtención de recursos financieros entrega una muestra real de lo valiosa que resulta una estrategia conjunta. En el caso que se comenta, la búsqueda conjunta de donaciones rindió mejores resultados para los integrantes que el trato directo de cada uno con los posibles donantes, de modo que se logró triplicar los aportes individuales anteriores. Las estrategias sistémicas son, además, un elemento importante en la planificación académica y, con ellas, se logró una reducción de la oferta académica superflua de programas, posibilitando la reinversión de recursos, siempre limitados, en áreas de alta prioridad. De este modo, se eliminaron o reconfiguraron más de cien programas, cantidad nunca antes alcanzada de manera individual.

El factor "eficiencia" puede argumentarse simplemente como la demostración de que un conjunto de instituciones es más eficiente que cada una operando independientemente. Sin embargo, no es fácil tomar conciencia de esta potencialidad, tampoco actualizarla y demostrarla, y es poco probable que un grupo de instituciones autónomas adopte, espontáneamente, una acción concertada de cooperación. Darse cuenta del potencial del sistema en términos de su eficiencia requiere un diseño creador y un decisivo liderazgo capaz de neutralizar las fuerzas centrífugas que caracterizan a la mayoría de los sistemas. Se suele apreciar su eficiencia como antagónica con la autonomía institucional, pues la mayoría de las entidades pretende ofertar la serie completa de servicios administrativos. El UMS exploró y concretó la posibilidad de crear centros comunes de servicio para operaciones administrativas, tales como adquisiciones, administración de recursos humanos, salud y seguridad, entre otras, teniendo como consecuencia reducción de costos, mejora en la calidad del servicio y una menor burocracia central. 
El factor de "responsabilidad social" o accountability, es clave en esta década y en la recién pasada. Quienes pagan la universidad, los legisladores, los contribuyentes, las agencias estatales que aportan financiamiento y los donantes privados, exigen que los recursos proporcionados sean utilizados correctamente. Podría estimarse que, por tratarse de procedimientos de relativa complejidad y dimensión, no se aplicaría a las universidades tal demanda. Es cierto que las burocracias podrían ampliar la brecha entre consumidor y proveedor de modo de disminuir esa responsabilidad; sin embargo, los sistemas no generan tales brechas y, por el contrario, aquéllos verdaderamente efectivos facilitan las relaciones internas adecuadas intentando que la responsabilidad social no quede encerrada en los campi. Su acción principal está en garantizar que su enlace con la autoridad se dé en el nivel adecuado y no en establecer decisiones compulsivas, sino recomendaciones respecto de temas críticos, como los niveles específicos de enseñanza o las matrículas.

El factor "integridad" apunta a la administración central encargada de la supervisión interna y externa. La primera asegura la responsabilidad social y la segunda, la integridad institucional correspondiéndole cautelar las intervenciones indebidas en las operaciones de cada uno de los integrantes. La estructura del sistema genera su natural defensa frente a posibles intenciones externas de establecer o intervenir en sus planes, programas o proyectos.

Sinergia, estrategia, eficiencia, responsabilidad social e integridad son las características que producen una eficaz, eficiente y efectiva cooperación; se constituyen, al mismo tiempo, en los parámetros según los cuales los sistemas han de ser evaluados y juzgados y en los puntos neurálgicos a través de los cuales se puede agregar valor a la empresa educativa.

Los factores de protección y los factores de riesgo

Para que las acciones de cooperación y los intercambios generen impacto y resultados positivos es necesario formular, y luego implementar, oportunidades nacionales e internacionales que desafíen con exigencias altas a académicos y estudiantes, actores centrales en toda universidad. Anderson (1999) propone un conjunto de variables 
-posibles de denominar como "factores de protección" y "factores de riesgo"- que permiten diseñar y ejecutar la cooperación universitaria y, a su vez, evaluar y validar la calidad y excelencia por parte de las propias instituciones concurrentes.

Entre los factores de protección que colaboran en alcanzar un programa de colaboración de calidad y con buenos productos, se menciona:

- Existencia de un apoyo "fuerte, claro y firme" desde las autoridades superiores.

- Convencimiento, por parte de los participantes, de que tienen iguales oportunidades, deberes y derechos.

- Presencia de quienes adoptan las decisiones claves pertinentes en los niveles correspondientes.

- Existencia de modelos de evaluación crítica y del esfuerzo por mantenerlos en el tiempo.

- Buena difusión, ojalá antes de su inicio.

- Procesos de decisión claramente delineados y comprensibles desde el inicio hasta la conclusión del programa.

- Claridad en las líneas de autoridad durante su ejecución, así como la fluidez y disponibilidad de los recursos humanos, financieros y físicos comprometidos durante el período de tiempo determinado para comenzar y probar el proyecto.

- Conocimiento detallado de las condiciones con que operan los subsidios externos involucrados.

- Permanencia de los términos del acuerdo entre todos los participantes durante el período de ejecución establecido.

Los "factores de riesgo" identificados se relacionan con:

- Ausencia del componente de liderazgo, fundamental en estos procesos en los que se requieren personas con experiencia práctica, con perspectiva y conocimiento internacional respecto de los países que participan, con capacidad para relacionarse con equipos profesionales y técnicos involucrados, con talento administrativo y con destrezas básicas en el uso de las tecnologías adecuadas.

- Inexistencia de seguros que respalden posibles emergencias. 
- No término oportuno de programas que no estén operando.

- Ausencia de correcciones y ajustes oportunos de los posibles errores en la planeación y conducción de iniciativas de cooperación internacional.

- Intervención en programas no congruentes con la misión fundamental de cualquiera de los participantes individuales, o del grupo colaborador como un todo.

- Existencia de mercados reducidos.

- Programas que sólo tengan impacto en el corto plazo.

- Generación de expectativas sobredimensionadas y el no estar alerta a tal posibilidad.

- Inexistencia de equipos humanos ajustados o complementados unos con otros, cuestión para la cual es relevante un conocimiento previo profundo, logrado, quizás, a través de las acreditaciones vigentes.

Algunas consideraciones respecto de la cooperación universitaria actual en Chile

En cuanto a la movilidad estudiantil en nuestro país, una prioridad importante debiera otorgársele al cambio y ajuste de los esquemas educacionales que se aplican en algunas universidades. Se requiere avanzar, gradualmente, hacia otras modalidades un tanto más flexibles, que faciliten la transferencia y movilidad de los alumnos, tanto al interior de las instituciones como entre ellas, contribuyendo así a la diseminación sistemática del conocimiento.

Es paradojal, pero con frecuencia resulta más fácil el intercambio internacional que el nacional. Esto se debe a que en Chile, prácticamente, no existen en el nivel de pregrado programas de intercambio de alumnos, a pesar de que el esfuerzo económico que representa la salida al extranjero, en la mayoría de los casos, es absorbido por los propios estudiantes o sus familias. Los ciclos de bachilleratos que existen en algunas universidades facilitan la movilidad estudiantil pero ésta no sólo hay que referirla a los posibles cambios de carrera-como habitualmente suele entenderse-, sino que debiera incluir una real ampliación de las oportunidades de estudio. También, el que la cooperación esté basada en el reconocimiento y 
confianza mutuos entre las instituciones desemboca en una ayuda eficiente.

Un tanto distinta es la situación en el nivel de posgrado, especialmente en los doctorados. Existen programas patrocinados y ejecutados en conjunto por varias universidades y, al respecto, se han dado señales claras. Un ejemplo lo constituye el MECESUP, que incentiva y respalda, con recursos financieros, acciones de cooperación nacional que ya han recibido apoyos académicos, por ejemplo: las acreditaciones positivas emitidas por la Comisión Nacional de Acreditación de Pregrado (CONAP). Interesa señalar la creación de redes nacionales de doctorados que ya existen en cinco áreas del conocimiento en las que participan ocho universidades. Por otra parte, un $9 \%$ de los doctorados hoy vigentes en Chile, corresponden a casos de cooperación nacional y, desde luego, casi la totalidad de los ciento veinticuatro programas existentes cuentan con convenios de apoyo internacional. Hay, además, otros once programas desarrollados en conjunto por instituciones nacionales y extranjeras, en los cuales la entrega del grado académico, en ocasiones, corresponde a la entidad extranjera y, en otras, se realiza en conjunto.

Interesa señalar que, en el área de la investigación, se aprecia una tendencia al incremento de las acciones de cooperación, en especial las de carácter internacional. La promoción e incentivo a la creación y consolidación de redes y de consorcios, constituyen señales claras y precisas que han sido bien acogidas por las instituciones. No es posible, aún, efectuar un análisis profundo de lo sucedido en este tema en el país. Si se toma, por ejemplo, el Concurso Regular del Fondo Nacional de Desarrollo Científico y Tecnológico (FONDECYT), cuyas condiciones de desarrollo no establecen como requisito acciones con carácter de cooperación -lo que significa que ésta se ha asumido con libertad y espontaneidad- los resultados tienen cierto interés. El gráfico adjunto recoge los porcentajes de proyectos ganadores en cada concurso con carácter de colaboración, sea éste de instituciones sólo nacionales o de nacionales con extranjeras. Al analizar la evolución de esos porcentajes en el tiempo, no es posible identificar una tendencia clara en términos de cooperación. Se tienen puntos altos y bajos en rangos muy alejados, entre el $0 \%$ y el 27\% para la cooperación universitaria y entre el $0 \%$ y el $26 \%$ para la cooperación de las 
universidades con otras entidades no universitarias. En todo caso, el promedio de proyectos asociados en todo el período de acción del FONDECYT está cerca del $11 \%$ del total si se toma en cuenta sólo la asociación con otras universidades y del 20 \% si además se incluyen instituciones no universitarias.

Concurso Regular FONDECYT, período 1982-2003

Proyectos aprobados y realizados en acciones cooperativas (porcentajes sobre el total de proyectos aprobados)

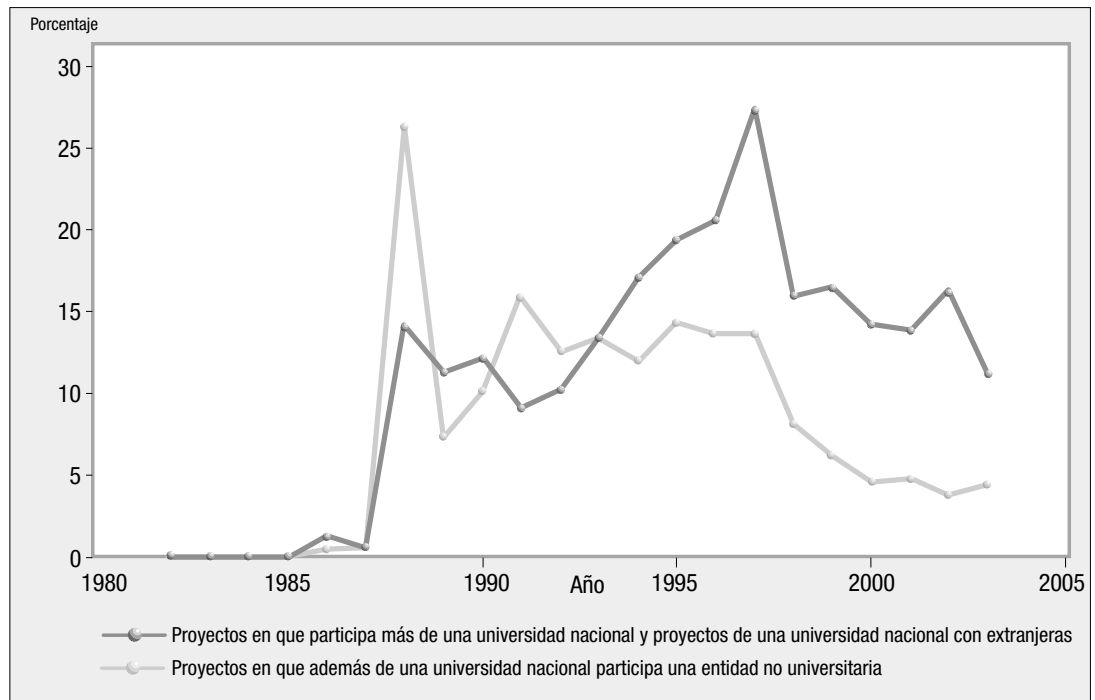

Finalmente, es evidente que, tal como se señalara anteriormente, la diversidad de instituciones universitarias es una condición necesaria para la cooperación que también se aplica al caso chileno. Ciertamente, nuestro país requiere de un sistema de evaluación de acuerdo con estándares de aceptación general para avanzar hacia una mayor colaboración universitaria. Es también indispensable que los instrumentos de certificación de la calidad no desincentiven la heterogeneidad del sistema universitario ya que, con ello, se estaría eliminando uno de los elementos más fuertes para la cooperación. No se puede olvidar que, en la homogeneidad, resulta más conveniente la acción individual que la cooperativa. 


\section{REFERENCIAS BiBLIOGRÁFICAS}

Anderson, Wayne, Cooperating Internationally, en New Directions for Higher Education, No 106, Summer 1999, pp. 101-7.

Fermoso, Julio, University Co-Operation between Europe and Latin America, en European Journal of Education, Vol. 28, N 1, 1993, pp. 85-98.

Fincher, Mark, Private University and Community College Strategic Alliances: The Case for Cooperation, en Community College Journal of Research and Practice, 26, 2002, pp. 349-61.

Hagen, Jon, University Co-operation and Academic Recognition in Europe: the Council of Europe and the Communities, en European Journal of Education, Vol. 22, No 1, 1987, pp. 77-83.

Langenberg, Donald, Why a system?, en Change, Mar/Apr 1994, Vol. 26, Issue 2, pp. 8-9.

Llano, Alejandro, El Futuro de la Libertad, Eunsa, Pamplona, 1985, pp. 137-8.

Reed, Charles B., Building Bridges across our Education System, en Thrust for Educational Leadership, May/June 2000, Vol. 29 Issue 5, pp. 20-1.

Rosenzweig, Robert M., Universities Change, Core Values Should Not, en Issues in Sciences and Technology, Winter 1999/2000, Vol. 16 Issue 2, pp. 59-64.

Simonyi, Agnes, The Evaluation of University-Region Relationships, en European Journal of Education, September 1999, Vol. 34 Issue 3, pp. 335-41. 
